\title{
Technical feasibility of a care management system for follow-up of patients hospitalized for acute coronary syndrome
}

\author{
Martin Juneau ${ }^{1}$, Malorie Chabot-Blanchet ${ }^{2}$, Sylvie Cossette ${ }^{3}$, Marie-Claude Guertin $^{2}$ and Robert F. DeBusk $^{* 4}$ \\ ${ }^{1}$ Professor of Medicine, Faculty of Medicine, University of Montreal, Director, Prevention Division, Montreal Heart Institute, Montreal, Canada \\ ${ }^{2}$ Montreal Heart Institute Rresearch Centre, Montreal, Canada \\ ${ }^{3}$ Montreal Heart Institute, Montreal, Canada \\ ${ }^{4}$ Professor of Medicine, Emeritus, Division of General Medical Disciplines, Department of Medicine, Stanford University School of Medicine, Stanford, California
}

\begin{abstract}
Objective: To evaluate the clinical feasibility of a telephone-based Care Management System (CMS) staffed by a nurse and cardiologist in coordinating the care of patients reporting cardiovascular symptoms following an acute coronary syndrome.

Background: Patients with established coronary artery disease (CAD) experiencing chest pain can be reliably risk-classified by telephone.

Methods: Randomized controlled trial. Treatment patients completing this randomized controlled trial ( $\mathrm{n}=90)$ were provided with clinic-hours telephone access to CMS, whereas patients receiving only usual care $(n=84)$ were not. The CMS nurse used a standardized telephone algorithm to translate patients' symptoms into provisional diagnoses and risk categories, for immediate telephone review by the CMS cardiologist. High-risk patients were advised to call 911 for transport to the ED, moderate-risk patients were advised to undergo a same-day clinic visit with the CMS cardiologist and low-risk patients were advised to stay at home, pending a followup telephone contact by CMS staff.
\end{abstract}

Results: The proportion of ED visits classified at the conclusion of the study as « urgent/ emergent » vs. " non-urgent/emergent » was greater in the treatment group than in the usual care group: 27 of $38(71 \%)$ vs. 8 of $25(32 \%)$ visits, respectively (p=0.02). In response to 21 patient-initiated telephone reports of chest pain, CMS advised staying home (7 cases), a same-day clinic visit (8 cases) or an ED visit (6 cases). No patient experienced adverse effects of participation.

Conclusions: Clinic-hours telephone triage for patients with recent ACS substitutes an expedited outpatient evaluation for an ED visit in most cases.

\begin{abstract}
Abbreviations and definitions: ACC/AHA: American College of Cardiology/ American Heart Association; Acute MI: Acute Myocardial Infarction; ED: Emergency Department; CMS: Care Management System; ACS: Acute Coronary Syndrome; ECG: electrocardiogram; STEMI: ST-segment elevation myocardial infarction; Non-STEMI: non-ST segment elevation myocardial infarction; PCI: Percutaneous coronary intervention
\end{abstract}

\section{Introduction}

Among the 8 million Americans undergoing ED evaluation for chest pain annually, half are hospitalized [1], yet only $20 \%$ are found to have an acute myocardial infarction (MI) [2]. Accordingly, the healthcare system processes 100 patients with chest pain through the ED and 50 through the hospital to identify the 10 with heart attack, at an annual cost of approximately $\$ 40$ billion [3]. The objective of the present study was to explore the feasibility of alternatives to an automatic ED visit for patients classified by telephone as low or moderate risk according to algorithms developed by the American College of Cardiology (ACC) and the American Heart Association (AHA) [4]. A Care Management System, CMS, developed by the Stanford Cardiac Rehabilitation Program, was used by the principal author, MJ, a cardiologist, and a team of nurses to coordinate the management of patients who contacted the Montreal Heart Institute (MHI) call center. The study was designed to document the clinical processes and outcomes of care provided to patients hospitalized for ACS, who were subsequently randomized to receive usual care only or telephone triage and care coordination in addition to usual care.

\section{Methods}

Between October 2008 and February 2010, all patients hospitalized with documented ACS at the Montreal Heart Institute or Centre Hospitalier Pierre Boucher were screened by study nurses. Included were patients with standard ECG and enzymatic criteria for STsegment elevation myocardial infarction (STEMI), non-ST segment elevation myocardial infarction (non-STEMI) and unstable angina. Exclusion criteria included incapacitating or life-threatening medical comorbidity, psychiatric conditions and inability to attend sameday cardiology clinic visits due to residence more than 50 miles from the enrolling center. The study was approved by the cognizant ethics committees. Patients in both study groups were telephoned

Correspondence to: Robert F. DeBusk, M.D., Department of Medicine, Stanford University School of Medicine, Stanford, California Email: Debusk@stanford.edu

Received: November 21, 2016; Accepted: December 12, 2016; Published: December 15, 2016 
monthly regarding cardiovascular symptoms, clinic and ED visits, hospitalizations and medications. The study protocol was presented to and endorsed by the staff cardiologists at the two participating medical centers. Study nurses were trained by Stanford-based staff in the use of the online CMS database application, including the use of diagnostic algorithms for triage.

Patients were randomized by study staff who used a standardized randomization table. Patients randomized to the treatment group were provided with toll-free telephone access to the study call center during clinic hours, Monday-Friday, 8:30 AM to 4:30 PM and were advised to call 911 for after-hours concerns. CMS nurses had immediate telephone or in-person access to the CMS cardiologist during clinic hours. The algorithm used for risk stratification was based on ACC/AHA guidelines for suspected ACS [4]. Based upon their risk category, patients were advised by the CMS team to: 1) Call 911 for transport to the nearest ED. Patients' personal cardiologists were apprised of this disposition by telephone. 2) Attend a same-day clinic visit with the CMS cardiologist or their personal cardiologist. CMS nurses telephoned cardiology clinic staff to apprise them of the patient's imminent arrival and to request an expedited ECG recording. Measurement of cardiac biomarkers and additional risk stratification procedures were performed on the same or the following day, at the discretion of the CMS cardiologist. 3). Remain at home, pending a follow-up telephone contact from the CMS nurse in 24-72 hours, depending on the day of the week.

Adjudication of ED visits as "urgent/emergent" or "non-urgent/ emergent" and corroboration of patients' monthly reports of clinical events were performed at the conclusion of the study by two independent cardiologists blind to the study group assignment who reviewed a clinical summary of the ED notes created by a research associate. The clinical summary incorporated the patient's chief complaint(s), pertinent historical and physical findings, the results of any specialized tests, the admitting and discharge diagnoses and any treatments provided during the ED visit. The clinical summary did not identify any healthcare professionals with whom the patient had interacted prior to the $\mathrm{ED}$ visit. $\mathrm{ED}$ visits requiring an urgent/emergent medical response were defined by the National Hospital Ambulatory Medical Care Survey (NHAMCS) criteria as those in which "The patient requires immediate attention for an acute illness or injury that threatens life or function and where delay would be harmful" [5]. Nonurgent/emergent visits were defined as those in which "the patient does not require attention immediately or within a few hours" [6,7]. The primary study endpoint was the proportion of ED visits classified as urgent/emergent. We hypothesized that the proportion of such visits could be increased by coordination of care by CMS staff. Sample size calculation was based on a comparison of the proportion of urgent/ emergent ED visits between the two groups, using visits as the unit of analysis. A review of the medical records of 100 consecutive patients discharged from the CCU at Montreal Heart Institute in the year prior to the present study disclosed 31 patients who made one or more ED visits during the following 6 months-a total of 67 ED visits. Among these 67 visits, 18 or $27 \%$ resulted in CCU admission and were judged urgent/emergent, while 49 or $73 \%$ resulted in discharge home and were judged non-urgent/emergent. Based on the proportion of ED visits judged urgent/emergent, we calculated that a sample size of 152 patients (76 patients per group) was needed to detect an absolute difference of $36.5 \%$ in the proportion of such ED visits between the two groups, with a power of $90 \%$. To account for an estimated dropout rate of $15 \%$, the number of patients per group was increased to 90 . The power calculation, based on an alpha error of $5 \%$, assumes that the number of ED visits per patient follows a quasi-Poisson distribution with a mean of 0.67 and variance of 1.34 i.e. twice the mean, to account for possible over-dispersion. An intra-class correlation coefficient of 0.3 was assumed. We used generalized estimating equations (GEE) to account for the possible correlation between visits within a patient and to account for two covariables: the availability of the intervention (CMS open or closed) and the reason for the ED visit (cardiac vs. non cardiac). Statistical analyses were performed using SAS 9.2 and significance level was defined as $\mathrm{p}<0.05$. Sample size calculation was based on the formula reported by Kang et al. [8].

\section{Results}

Reasons for exclusion are shown in Figure 1. Among the 1,369 patients screened for enrolment, 92 were randomized to the treatment group and 88 were randomized to the usual care group. Six patients were excluded from analysis: two in the treatment group who refused to continue participation and four in the usual care group, of whom 3 refused to continue and one did not fulfill the criteria for ACS. Consequently, analysis by modified intention to treat is based on 90 patients in the treatment group and 84 patients in the usual care group. The two study groups were well matched on baseline characteristics (Table 1).

Table 1. Baseline characteristics of patients.

\begin{tabular}{|c|c|c|c|}
\hline Parameters & $\begin{array}{l}\text { Usual Care } \\
\text { Group } \\
(\mathbf{n}=\mathbf{8 4})\end{array}$ & $\begin{array}{l}\text { Treatment } \\
\text { Group } \\
(\mathbf{n}=90)\end{array}$ & P value \\
\hline Age (years) & $61 \pm 11$ & $61 \pm 10$ & 0.65 \\
\hline Men (n and \%) & $65(77 \%)$ & $77(86 \%)$ & 0.16 \\
\hline First Acute Coronary Syndrome & $46(55 \%)$ & $36(40 \%)$ & 0.05 \\
\hline History of prior angina & $52(62 \%)$ & $54(60 \%)$ & 0.80 \\
\hline History of prior Coronary surgery & $12(14 \%)$ & $14(16 \%)$ & 0.81 \\
\hline History of prior heart failure & $4(5 \%)$ & $3(3 \%)$ & 0.63 \\
\hline History of prior stroke & $1(1 \%)$ & $2(2 \%)$ & 0.60 \\
\hline History of claudication & $9(11 \%)$ & $15(17 \%)$ & 0.26 \\
\hline \multicolumn{4}{|l|}{ Risk factors } \\
\hline High blood pressure & $51(61 \%)$ & $49(54 \%)$ & 0.40 \\
\hline Hypercholesterolemia & $69(82 \%)$ & $73(81 \%)$ & 0.86 \\
\hline Current smokers & $23(27 \%)$ & $27(30 \%)$ & 0.70 \\
\hline Diabetes & $13(15 \%)$ & $18(20 \%)$ & 0.44 \\
\hline \multicolumn{4}{|l|}{ Risk status } \\
\hline Low & $32(38 \%)$ & $22(24 \%)$ & \\
\hline Moderate & $36(43 \%)$ & $49(54 \%)$ & 0.14 \\
\hline High & $16(19 \%)$ & $19(21 \%)$ & \\
\hline \multicolumn{4}{|c|}{ Discharge diagnosis at randomization } \\
\hline Unstable angina & $30(36 \%)$ & $32(36 \%)$ & \\
\hline $\begin{array}{l}\text { Non-ST segment elevation } \\
\text { myocardial infarction }\end{array}$ & $31(37 \%)$ & $22(24 \%)$ & 0.12 \\
\hline $\begin{array}{l}\text { ST segment elevation myocardial } \\
\text { infarction }\end{array}$ & $23(27 \%)$ & $36(40 \%)$ & \\
\hline $\begin{array}{l}\text { Left ventricular ejection fraction } \\
(\%, \pm \text { SD })\end{array}$ & $54 \pm 11$ & $54 \pm 9$ & 0.69 \\
\hline Coronary angiogram & $76(90 \%)$ & $85(94 \%)$ & 0.32 \\
\hline $\begin{array}{l}\text { Percutaneous coronary } \\
\text { intervention/stent }\end{array}$ & $71(85 \%)$ & $82(91 \%)$ & 0.18 \\
\hline \multicolumn{4}{|l|}{ Medications ( $\mathrm{n}$ and \%) } \\
\hline ACE inhibitors & $38(45 \%)$ & $51(57 \%)$ & 0.13 \\
\hline Angiotensin II receptor blockers & $18(21 \%)$ & $26(29 \%)$ & 0.26 \\
\hline Beta blockers & $75(89 \%)$ & $71(79 \%)$ & 0.06 \\
\hline Calcium antagonists & $20(24 \%)$ & $26(29 \%)$ & 0.45 \\
\hline Aspirin & $82(98 \%)$ & $88(98 \%)$ & 0.94 \\
\hline Other Antiplatelet Agents & $76(90 \%)$ & $80(89 \%)$ & 0.73 \\
\hline Statins & $82(98 \%)$ & $86(96 \%)$ & 0.46 \\
\hline Nitrates & $13(15 \%)$ & $10(11 \%)$ & 0.40 \\
\hline Anticoagulant (Warfarin) & $11(13 \%)$ & $11(12 \%)$ & 0.86 \\
\hline
\end{tabular}




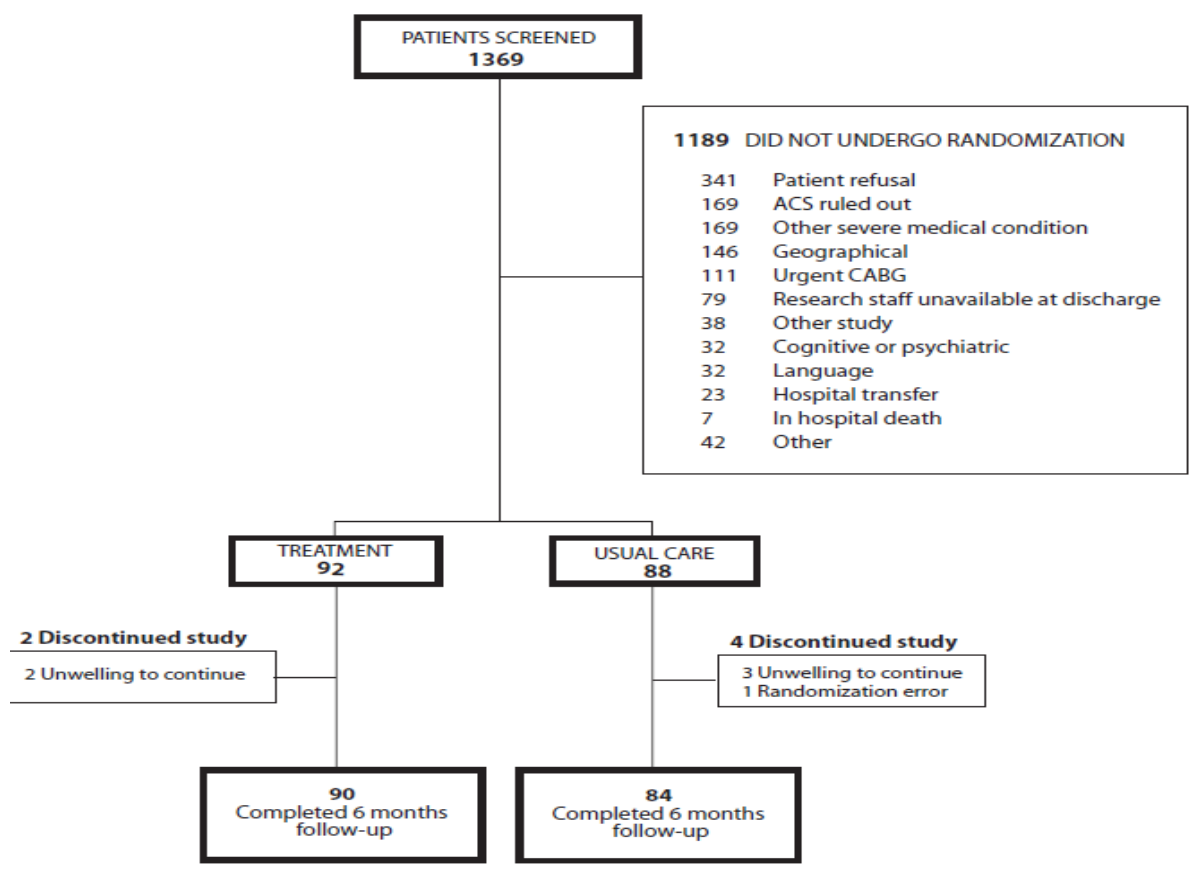

Figure 1. Flow of Participants. ACS: Acute Coronary Syndrome, CABG: Coronary Artery Bypass Graft.

\section{Emergency Department Visits: \\ Treatment Group $(n=90)$}

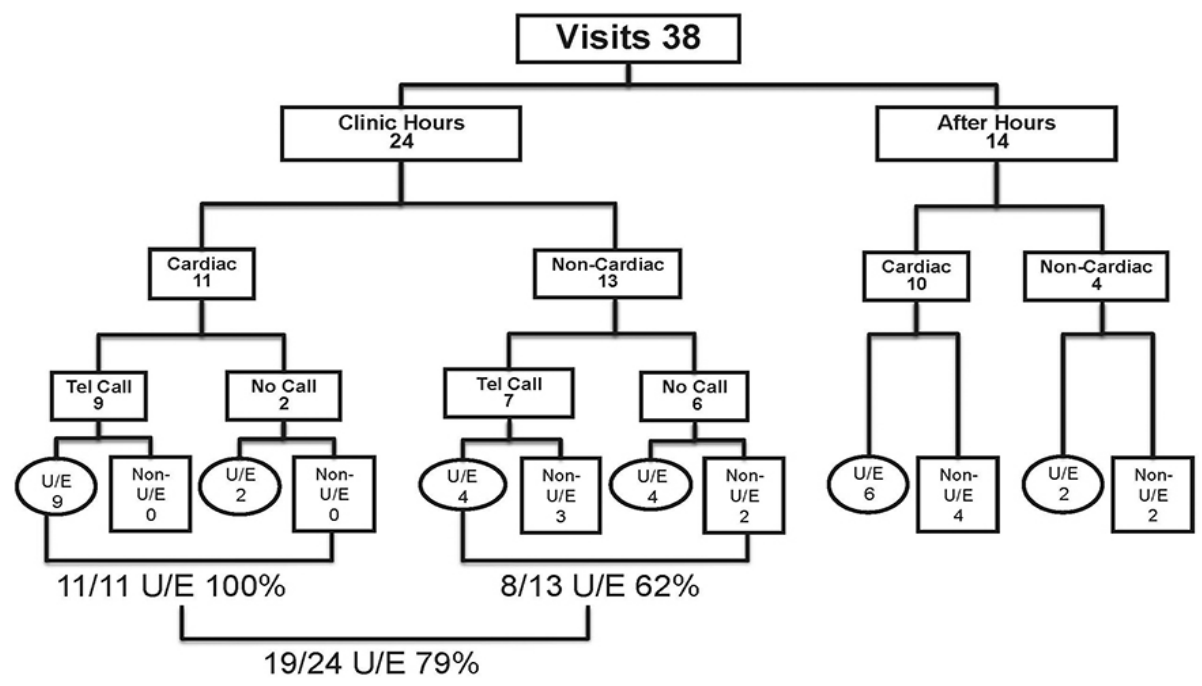

Figure 2. Emergency Department Visits: Treatment Group. Tel: Telephone, U/E: Urgent/emergent, non-U/E: non-urgent/emergent

There were no deaths or acute myocardial infarctions (MIs). Six patients in each group met the ACC/AHA criterion of unstable angina [4]. Six patients in the treatment group underwent coronary angioplasty and stenting for ACS. Four others hospitalized for atypical chest pain received medical therapy. Five patients in the usual care group underwent coronary angioplasty and stenting for ACS. One patient hospitalized by his personal cardiologist for atypical chest pain received medical treatment following coronary angiography.

The total number and the reasons for cardiac and non-cardiac ED visits that occurred during or after clinic hours and the adjudication of the ED visits as urgent/emergent or non-urgent/emergent are shown in Figure 2 for the treatment group and in Figure 3 for the usual care group.

The proportion of ED visits classified as urgent/emergent was 2.3 times greater in the intervention group than in the usual care group: among the $38 \mathrm{ED}$ visits in the treatment group, 27 (71\%) were classified as urgent/emergent compared to 8 of $25 \mathrm{ED}$ visits in the usual care group (33\%) ( $\mathrm{P}=0.02)$. During clinic hours, the proportion of urgent/ emergent ED visits was 3.2 times greater in the intervention group than in the usual care group: among the 24 clinic-hours ED visits made by treatment patients, 19 (79\%) were classified as urgent/emergent compared to 4 of 16 clinic-hours ED visits (25\%) made by usual care 


\section{Emergency Department Visits: Usual Care Group $(n=84)$}

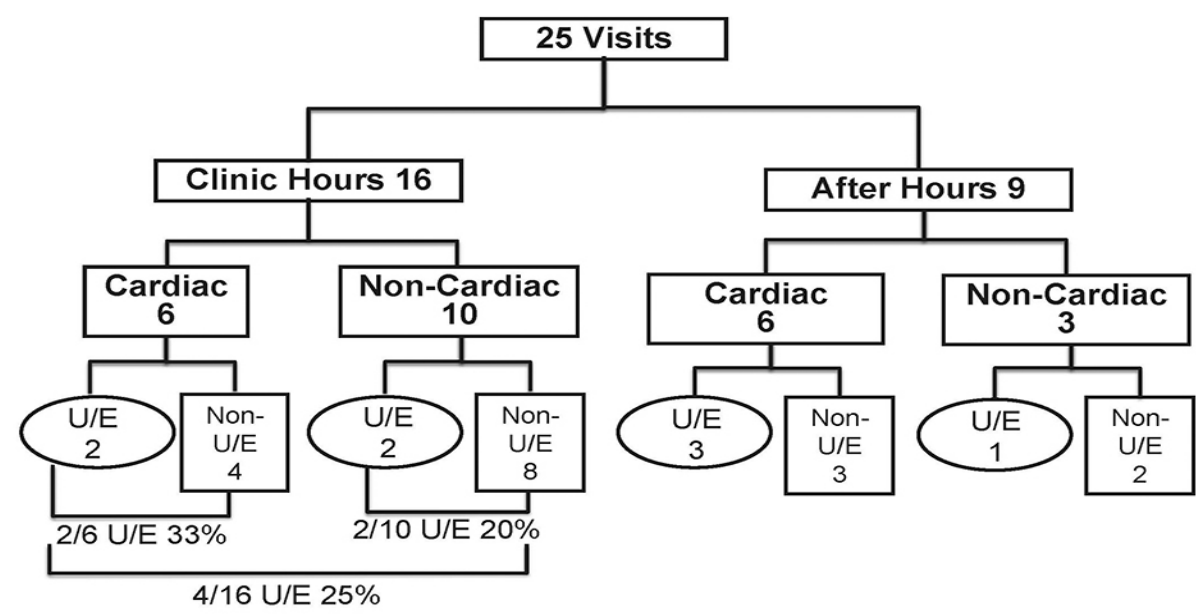

Figure 3. Emergency Department Visits: Usual Care Group U/E: Urgent/emergent, non-U/E: non-urgent/emergent.

\section{Patient-Initiated Call Center Contacts}

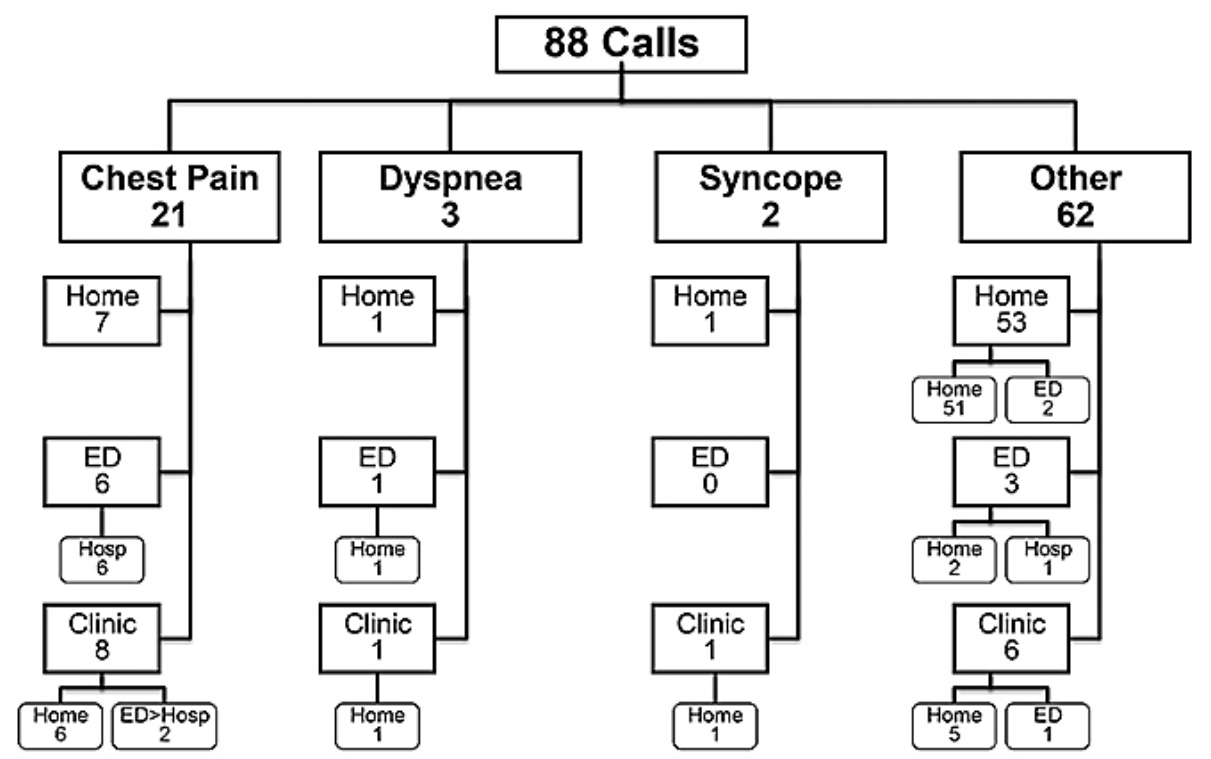

Figure 4. Patient-Initiated Call Center Contacts. ED: Emergency Department, Hosp: Hospital.

patients $(\mathrm{p}=0.0075)$. The proportion of urgent/emergent ED visits for cardiac complaints was 17 of 21 ED visits $(81 \%)$ in the treatment group and 5 of 13 ED visits (38\%) in the usual care group ( $\mathrm{p}=0.03)$.

As shown in Figure 2, all eleven ED visits for cardiac reasons in the treatment group were classified as urgent/emergent. In 8 cases, the ED visit was preceded by a patient-initiated telephone contact and in one case during a scheduled telephone contact initiated by CMS staff. ED evaluations of chest pain in two patients, who were referred by their physicians, without prior contact with CMS, were classified as urgent/ emergent; both patients were admitted to the CCU and underwent coronary angiography and PCI. Seven ED visits for non-cardiac reasons were preceded by an inbound call (Figure 3), of which 4 were judged urgent/emergent and 3 were judged non-urgent/emergent.

The proportion of urgent/emergent after-hours ED visits was comparable: in the treatment group, 6 of 10 after-hours ED visits for cardiac symptoms were judged urgent/emergent (Figure 2), compared to 3 of 6 after-hours ED visits for cardiac symptoms in the usual care group, (Figure 3). A similar pattern was noted for patients reporting non-cardiac symptoms. The ED visits independently classified by the two cardiologists as urgent/emergent agreed in all cases.

The advice provided to patients calling to report chest pain was 
consistent with the ACC/AHA algorithm in all but two cases. The CMS cardiologist advised one patient to undergo a same-day clinic visit when the algorithmic advice was to stay home. The patient's antianginal medication was increased and coronary artery bypass graft surgery was performed three days later. The CMS nurse advised one patient to go directly to the ED when the algorithmic advice was for the patient to stay home. The patient was admitted to the CCU for assessment of atypical chest pain and discharged following a negative troponin test and a negative stress echocardiogram.

Patients concurred with the advice provided by the CMS nurse in 13 of 16 (81\%) calls. The 3 patients who went directly to the ED were discharged home, following evaluation of hypertension in 2 cases and an allergic reaction in one case. Four patients experiencing non-cardiac symptoms went directly to the ED during clinic hours without calling CMS; visits for a suicidal attempt and physical trauma were judged urgent/emergent and visits for anxiety attack and fatigue were judged non-urgent/emergent.

A total of 88 telephone contacts were initiated by 43 patients during the study: a single contact in 21 cases, 2 contacts in 12 cases and 3 or more contacts in 10 cases. Figure 4 shows that 26 of these 88 contacts (30\%) were for cardiac symptoms: 21 for chest pain, 3 for shortness of breath and 2 for syncope. The 7 patients reporting chest pain who were advised to stay home were asymptomatic during the CMS nurse's followup telephone contact; none experienced recurrent chest pain or visited their cardiologist within the following 2 weeks. All 6 patients advised to visit the ED immediately were hospitalized. Eight patients were scheduled for a same day clinic visit; an ECG and point-of-service troponin blood test were performed in all; a treadmill test was performed in 5 cases. Two patients were sent to the ED, one for suspected ACS and one for severe hypertension. The remaining 6 patients were sent home following evaluation of a variety of chronic medical conditions. The remaining $70 \%$ of inbound calls concerned medications $(n=22)$, non-cardiac symptoms $(n=21)$, and reporting a test result, an ED visit or hospital admission, or scheduling a routine follow-up clinic visit $(n=19)$.

\section{Discussion}

The present study demonstrates the clinical feasibility of a telephone-based triage system in which triage nurses used ACC/AHA algorithms for suspected ACS to establish a provisional diagnosis based on patients' reports of symptoms. CMS helped to streamline and systematize the process of triage and care coordination for patients reporting symptoms. CMS triage nurses promptly translated patients' reports of symptoms into provisional diagnoses and risk categories prior to consulting with the CMS cardiologist by telephone. In selected cases, the CMS cardiologist then consulted with the patient's customary physician regarding an appropriate clinical disposition, which was implemented by the CMS nurse and communicated to the patient by telephone. A report summarizing the telephonic interactions among patients and physicians was then distributed to the patient's personal physician.

Prompt risk stratification permits uncoupling of two essential elements of the care process: initial establishment of a risk category and subsequent provision of definitive care. For patients classified as high-risk, an immediate ED evaluation is imperative. Patients' reports of chest pain, elicited by telephone [9], permit immediate identification of patients at high-risk for death or non-fatal MI, who stand to benefit most from percutaneous coronary interventions or surgery.
For patients classified as moderate risk, the evidence supporting an automatic ED visit is less clear. The ACC/AHA classification of "moderate risk" includes patients experiencing new-onset angina during the preceding two weeks that restricts walking to 1-2 blocks or climbing one flight of stairs [4]. Persistence of anginal symptoms over a period as long as two weeks suggests that many such patients could safely undergo a same-day clinic evaluation in lieu of an ED visit, as in the present study. However, conducting this evaluation in a standard outpatient clinic setting is often problematic, for cardiologists are often hard-pressed to meet the needs of their asymptomatic patients, much less those of patients reporting new or worsening symptoms [10]. Moreover, outpatient clinic and specialized laboratory testing slots are allocated weeks or months previously to patients undergoing routine follow-up [10]. Accordingly, outpatient chest pain units or stepdown units associated with the ED may be better equipped to provide monitoring and measurement of biomarkers to these patients, in lieu of a formal ED admission. The proportion of ED visits occurring during clinic hours was similar in both groups: 24 of $38(62 \%)$ in the treatment group and 16 of 25 (64\%) in the usual care group, respectively. This was unexpected, since clinic hours compose only $25 \%$ of the 168 hours per week during which patients are at risk for ACS. This finding highlights the opportunity to substitute a same-day outpatient evaluation for an automatic ED visit in patients classified as moderate risk

Among patients classified as low-risk, a scheduled outpatient clinic visit occurring within the 72 hours following the patient's initial report of symptoms is generally sufficient to assure continuity of care. A 72-hour hiatus between the provisional diagnosis and definitive care also serves the needs of patients reporting symptoms after-hours who are classified as low-risk. For example, patients classified as low risk at $6 \mathrm{PM}$ on a Friday evening could be scheduled for a routine clinic followup visit as early as the following Monday morning or as late as the following Monday evening.

In the present study, the total amount of clinical effort devoted to the clinical management of the 88 clinic-hours telephone contacts initiated by patients in the treatment group was approximately 10 minutes per call ( 880 minutes) or 15 hours in all. A total of $20 \%$ of these contacts were for cardiovascular symptoms. The time required by the triage nurses to establish a provisional diagnosis and risk category for these patients averaged 4-5 minutes. The time required by the CMS cardiologist to formulate a clinical disposition during telephone consultation with the triage nurse was 1-2 minutes. The brevity of the cardiologist's participation in triage and coordination of care for patients reporting cardiovascular symptoms helped to minimize any interruption of the cardiologist's ongoing inpatient or outpatient clinical activities. The time required for the triage nurse to convey the clinical disposition to the patient, implement the arrangements for followup and distribute a clinical summary of the telephone encounter to patients' physicians averaged $4-5$ minutes. A total of $80 \%$ of patient-initiated telephone contacts were to report non-cardiovascular symptoms, request a clinic visit or information about medications or prescriptions. The triage nurses spent approximately 10 minutes in response to these contacts, often without consultation with the cardiologist.

Many ED visits are prompted not by an urgent medical need, but by the absence of a prompt and reliable telephone consultation with patients' personal physicians that could address patients' concerns $\left({ }^{10}\right)$. Indeed, only a minority of all ED visits meet the criteria of "urgent/ emergent" specified in the annual report of ED visits published by NHAMCS [11]. The number of patients seeking ED care was greater in the treatment group than in the usual care group: $38 v s .25$, a difference 
not explained by the timing of these ED visits (during $v s$. after-hours) or the reason for such visits (cardiac vs. non-cardiac). It is possible that treatment patients were sensitized to initiate a call to CMS or an after-hours ED visit than those receiving usual care only. Indeed, the proportion of treatment patients undergoing an ED visit during clinic hours who were classified as urgent/emergent was greater than that of control patients who visited the ED during clinic hours: $79 \%$ vs. $25 \%$. A similar proportion of PCI in both groups and the absence of deaths or acute MI's in either group may reflect excellent clinical care or other factors, including the comparatively young age of patients in both groups (61 years).

In the present study, the costs of providing telephone triage and care coordination largely reflected the effort of the program cardiologist and triage nurses who provided telephone triage and care coordination. Research activities such as enrollment and orientation of patients to the study protocol and followup contact to assess clinical processes and outcomes were reimbursed separately. Reimbursement for clinical activities was allocated as follows: $\$ 600$ to the cardiologist for 3 hours' effort and $\$ 600$ to the triage nurses for 12 hours' effort. A key assumption underlying this model of reimbursement for clinical activities is that the nurses who provide telephone triage and care coordination are already engaged in the telephone management of patients with an established affiliation with the institution.

The effort required to implement CMS in clinical settings is highly scalable. For example, a 50-fold expansion of the caseload of enrolled patients, from 90 in the present study to as many as 4,500 and doubling of the duration of followup from 6 to 12 months would increase the total amount of time required for telephone triage and care coordination to approximately 1,500 hours annually. This represents approximately 300 hours of effort annually for one or more cardiologists and 1,200 hours of effort annually for one or more triage nurses. Based on the model presented above, this represents annual salary costs of $\$ 120,000$. This figure is within the means of many hospitals seeking to avoid Medicare penalties for unnecessary readmissions for suspected ACS [12] or to obtain Medicare incentive payments for provision of more cost-effective care [13]. Moreover, these salary costs may be less than those presently expended by healthcare providers who lack online decision support tools and detailed clinical protocols for coordinating the care of patients reporting cardiovascular symptoms by telephone.

Limitations of the study. The present study was conducted under idealized circumstances in which clinic-hours telephone surveillance and triage and hands-on clinical management by a healthcare team was continuously available during clinic hours and agreement of patients' cardiologists to adhere to the CMS clinical protocol was established prior to initiation of the study. The present study was designed to demonstrate the feasibility of CMS in coordinating the care of patients with established cardiovascular disease. Our findings lay the groundwork for future studies conducted in diverse clinical and geographical settings and patient populations.

\section{Acknowledgments}

The authors are grateful for the dedication and essential contributions of the following individuals: At Stanford, Nancy Houston Miller, R.N., BSN and Lynda Raby provided training and ongoing liaison to the Montreal-based research team concerning the implementation of the Care Management System. At Montreal, study nurses Marie Cournoyer, R.N., Heidi Claveau, R.N., Manon (OK) Bélanger, R.N. and Johanne Loyer, R.N., provided telephone surveillance and triage and care coordination to study participants.
Julie Lalongé was the study coordinator and data manager. Stéphanie Rochette created the manuscript. Sylvie Maillette, M.D., a cardiologist at CHPB and Anil Nigam, M.D., a cardiologist at MHI, corroborated patients' reports of clinical processes and outcomes, including adjudication of the urgency/emergency of ED visits.

\section{Sponsor}

The study was sponsored by Montreal Heart Institute Foundation, Montreal, Canada.

\section{References}

1. Pitts SRN, XuJB, BurtCW (2008) National Hospital Ambulatory Medical Care Survey: 2006 Emergency Department Sumary. Hyattsville MD.

2. Manini AF, Gisondi MA, van der Vlugt TM, Schreiber DH (2002) Adverse cardiac events in emergency department patients with chest pain six months after a negative inpatient evaluation for acute coronary syndrome. AcadEmerg Med 9:896-902.

3. HealthCare.Gov. Report to Congress:National Strategy for Quality Improvement in Healthcare Washington: US Department of Health and Human services. 2011.

4. Anderson JL, Adams CD, Antman EM, Bridges CR, Califf RM, et al. (2007) guidelines for the management of patients with unstable angina/non ST-elevation myocardial infarction: a report of the American College of Cardiology/American Heart Association Task Force on Practice Guidelines (Writing Committee to Revise the 2002 Guidelines for the Management of Patients With Unstable Angina/Non ST-Elevation Myocardial Infarction): developed in collaboration with the American College of Emergency Physicians, the Society for Cardiovascular Angiography and Interventions, and the Society of Thoracic Surgeons: endorsed by the American Association of Cardiovascular and Pulmonary Rehabilitation and the Society for Academic Emergency Medicine. Circulation 116(7):e148-304.

5. Schappert SM (1995) The urgency of visits to hospital emergency departments: data from the National Hospital Ambulatory Medical Care Survey (NHAMCS), 1992. Stat Bull MetropInsur Co.76:10-19.

6. Stussman BJ (1996) National Hospital Ambulatory Medical Care Survey: 1994 emergency department summary. Adv Data1-20. [Crossref]

7. McCaig LF, Stussman BJ (1997) National Hospital Ambulatory Medical Care Survey: 1996 emergency department summary. Adv Data 1-20. [Crossref]

8. Kang S-H, AhnCW,JungS-H (2003) Sample size calculation for dichotomous outcomes in cluster randomization trials with varying cluster size. Drug Information Journal 37:109-114.

9. DeBusk RF, Miller NH, Raby L (2010) Technical feasibility of an online decision support system for acute coronary syndromes. Circ Cardiovasc Qual Outcomes 3:694-700.

10. Schuur JD, Venkatesh AK (2012) The growing role of emergency departments in hospital admissions. $N$ Engl J Med 367: 391-393. [Crossref]

11. Bhuiya FA, Pitts SR, McCaig LF (2010) Emergency department visits for chest pain and abdominal pain: United States, 1999-2008. NCHS Data Brief 1-8. [Crossref]

12. Lawrence D (2009) Get out--and stay out. With readmission penalties on the horizon, CIOs are laying the groundwork for post-discharge solutions. Healthc Inform 26: 3032. [Crossref]

13. Kocher RP, Adashi EY (2011) Hospital readmissions and the Affordable Care Act: paying for coordinated quality care. JAMA 306: 1794-1795. [Crossref]

Copyright: (C2016 Juneau M. This is an open-access article distributed under the terms of the Creative Commons Attribution License, which permits unrestricted use, distribution, and reproduction in any medium, provided the original author and source are credited. 